\title{
Fabrication of Hollow Waveguides with Sacrificial Aluminum Cores
}

John P. Barber

johnpbarber@yahoo.com

Donald B. Conkey

Jeffrey Ryan Lee

Neal B. Hubbard

hubbard_neal@yahoo.com

Larry L. Howell

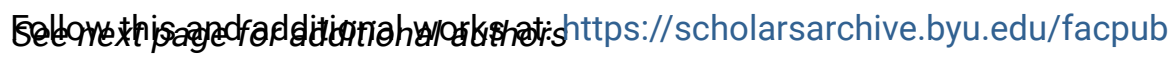

Part of the Mechanical Engineering Commons

\section{Original Publication Citation}

Barber, J.P., Conkey, D.B., Lee, J.R., Hubbard, N.B., Howell, L.L., Yin, D., Schmidt, H., and Hawkins, A.R., â€œFabrication of Hollow Waveguides with Sacrificial Aluminum Cores,â€ IEEE Photonics Technology Letters, Vol. 17, No. 2, pp. 363-365, 25.

\section{BYU ScholarsArchive Citation}

Barber, John P.; Conkey, Donald B.; Lee, Jeffrey Ryan; Hubbard, Neal B.; Howell, Larry L.; Hawkins, Aaron R.; Yin, Gongliang; and Schmidt, Holger, "Fabrication of Hollow Waveguides with Sacrificial Aluminum Cores" (2005). Faculty Publications. 394.

https://scholarsarchive.byu.edu/facpub/394

This Peer-Reviewed Article is brought to you for free and open access by BYU ScholarsArchive. It has been accepted for inclusion in Faculty Publications by an authorized administrator of BYU ScholarsArchive. For more information, please contact ellen_amatangelo@byu.edu. 


\section{Authors}

John P. Barber, Donald B. Conkey, Jeffrey Ryan Lee, Neal B. Hubbard, Larry L. Howell, Aaron R. Hawkins, Gongliang Yin, and Holger Schmidt 


\title{
Fabrication of Hollow Waveguides With Sacrificial Aluminum Cores
}

\author{
John P. Barber, Student Member, IEEE, Donald B. Conkey, Jeffrey Ryan Lee, Neal B. Hubbard, Larry L. Howell, \\ Dongliang Yin, Holger Schmidt, Member, IEEE, and Aaron R. Hawkins, Senior Member, IEEE
}

\begin{abstract}
We have developed a process to fabricate dielectric waveguide structures with long hollow cores formed by etching a sacrificial core material. The process is compatible with other planar silicon fabrication techniques. Using aluminum as the sacrificial material, we have investigated fabrication limits and design parameters that determine mechanical integrity of the waveguides. Internal pressure due to the production of gaseous compounds during the core removal process was identified as the yield-limiting factor. A mechanical model based on finite element analysis and confirmed by experiment, predicts ultimate pressures sustainable by these structures. Waveguides less than $10 \mu \mathrm{m}$ wide with $2-\mu \mathrm{m}$-thick coatings should sustain $50 \mathrm{MPa}$ of internal pressure. Low-loss guided-mode propagation in optical waveguides based on these hollow cores is demonstrated.
\end{abstract}

Index Terms-Fabrication, micromachining, photonic crystals, waveguides.

\section{INTRODUCTION}

C ONVENTIONAL optical waveguides confine light to a high-index medium and cannot be used to guide light in liquid or gaseous materials. Alternative methods include Bragg waveguides [1], Teflon light-pipes [2], and antiresonant reflecting optical waveguides (ARROWs). ARROW waveguides achieve guiding in low-index materials by using Fabry-Pérot reflectors to confine the light in a nonsolid core surrounded by a higher index medium [3], [4]. Fabrication of hollow ARROW waveguides, which can then be filled with liquid or gaseous media, allow new applications in biological sample analysis and other applications requiring interaction between light and low-index materials. Here, we report on the development of a method to fabricate hollow-core multilayer dielectric structures with micrometer dimensions and centimeter lengths applicable to multilayer waveguides such as ARROWs and photonic crystals.

Manuscript received July 26, 2004; revised September 28, 2004.

J. P. Barber, D. B. Conkey, J. R. Lee, and A. R. Hawkins are with the Electrical and Computer Engineering Department, Brigham Young University, Provo, UT 84602 USA (e-mail: jbarber@ee.byu.edu; dbc35@email.byu.edu; j1389@email.byu.edu; ahawkins@ee.byu.edu).

N. B. Hubbard and L. L. Howell are with the Mechanical Engineering Department, Brigham Young University, Provo, UT 84602 USA (e-mail: nealh@email.byu.edu; lhowell@byu.edu).

D. Yin and H. Schmidt are with the School of Engineering, University of California Santa Cruz, Santa Cruz, CA 95064 USA (e-mail: hschmidt@soe.ucsc.edu; yindl@soe.ucsc.edu).

Digital Object Identifier 10.1109/LPT.2004.839446

\section{FABRICATION METHOD}

Versatile hollow waveguides must meet several criteria. First they must be of reasonable length - usually at least $5 \mathrm{~mm}$ to allow for external connections to fluid channels. These structures must have smooth inner walls to prevent optical scatter or interruptions in fluid flow. Additionally, their fabrication method must be robust and construction times manageable if large networks of channels are to be made. It is also important that any fabrication method utilize standard complementary metal-oxide-semiconductor or microelectromechanical system techniques to take advantage of the economies of scale these processes offer. Current methods for making hollow multilayer waveguides can be classified into three categories all of which have been used in the past to make hollow channel structures: wafer bonding [5], self-sealing [6], and sacrificial core [7]. For this work, we have chosen the third construction method, surrounding a sacrificial core layer with material and then removing the core to reveal a hollow center. This allows for high fidelity definition of the core region by delineating the sacrificial layer using standard photolithography and etching.

Here we focus on ARROW waveguides that were built with alternating layers of silicon dioxide $(n=1.46)$ and silicon nitride $(n=2.00)$ and sacrificial cores. The dielectric layers were deposited using plasma-enhanced chemical vapor deposition (PECVD) at a temperature of $250{ }^{\circ} \mathrm{C}$ and pressure of 0.9 Torr. The growth rate was approximately $70 \AA / \mathrm{min}$ for nitride and $200 \AA / \mathrm{min}$ for oxide. PECVD layers were used because they are deposited conformally over sacrificial layers. We have fabricated ARROW waveguide structures using aluminum and polymers like SU8 that can be patterned with high fidelity and withstand the PECVD deposition temperatures.

The first step of the fabrication process is to deposit the bottom dielectric layers on a silicon wafer. Depending on the design wavelength of the waveguide, the layer thicknesses range from 100 to $350 \mathrm{~nm}$ [8], and a total of six layers are deposited. Depositing more layers results in better light guiding and six represents a compromise between ultimate performance and fabrication complexity. A sacrificial layer was then either evaporated (aluminum core) or spun (polymer core). Layer thicknesses were between 0.5 and $5 \mu \mathrm{m}$ and aluminum was patterned using either the liftoff method or wet etching.

The top six dielectric layers were deposited in the same manner as the bottom layers, with the thickness of the top layer increased to approximately $2 \mu \mathrm{m}$ to improve mechanical strength of the waveguide. Edges of the waveguides were then cleaved and the substrate placed in a selective etch $\left(\mathrm{HCl}: \mathrm{HNO}_{3}\right.$ 


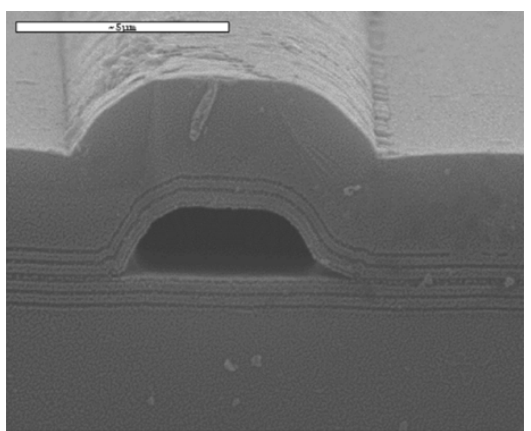

(a)

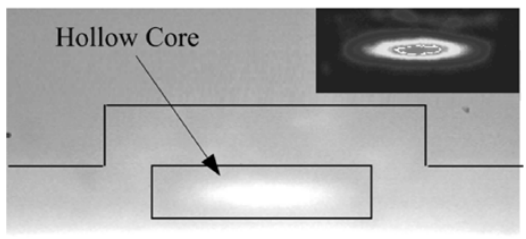

(b)

Fig. 1. (a) Cross-sectional SEM photograph of ARROW waveguide structure consisting of multiple layers of silicon nitride and silicon dioxide layers surrounding a hollow core. (b) Output facet image of mode propagating in hollow ARROW waveguide. Black lines: Outline of sample for clarity. The image in the upper right corner shows the intensity mode profile (near-field).

for $\mathrm{Al}, \mathrm{H}_{2} \mathrm{SO}_{4}: \mathrm{H}_{2} \mathrm{O}_{2}$ for polymers) to remove the sacrificial cores while leaving the dielectric layers intact. Fig. 1(a) shows a scanning electron microscope (SEM) image of a completed hollow ARROW waveguide formed using aluminum as the sacrificial layer. The shape of the opening is faithful to the original aluminum layer and comes from patterning the aluminum through liftoff resulting in the tapering sides seen in the picture. Optical mode guiding has now been measured in similar hollow core structures made using both aluminum and SU8 based sacrificial cores [3], [4]. Fig. 1(b) shows the mode image for $785-\mathrm{nm}$ light guided down a waveguide with a $12-\mu \mathrm{m}$ core width, 3.5- $\mu \mathrm{m}$ core height, and $2 \mathrm{~mm}$ length (SU8 core). The image was taken in the near field with a charged-coupled device camera. The black lines outline the facet of the waveguide. The optical mode (bright ellipse) is clearly confined inside the hollow air core. In the upper corner of the image, the intensity profile of the ARROW core mode is shown.

\section{FABRICATION LIMITS AND DESIGN PARAMETERS}

Although we have successfully made ARROWs using both aluminum and polymer sacrificial cores, this letter concentrates on the mechanical properties of those fabricated using aluminum cores. To do so, etch times and the nature of the etch process were investigated. Aluminum lines approximately $700 \mathrm{~nm}$ thick were patterned onto a silicon substrate. These lines varied in width between 10 and $300 \mu \mathrm{m}$. A single layer of silicon dioxide $3.0 \mu \mathrm{m}$ thick was then deposited over the aluminum, the silicon substrate cleaved, and then the samples placed in an aqua regia (3:1 mixture of hydrochloric and nitric acid) solution. Samples were periodically removed from the acid solution and the amount of aluminum that was etched was measured using an optical microscope. Fig. 2 shows a graph of the total length of aluminum etched versus time in the etchant.

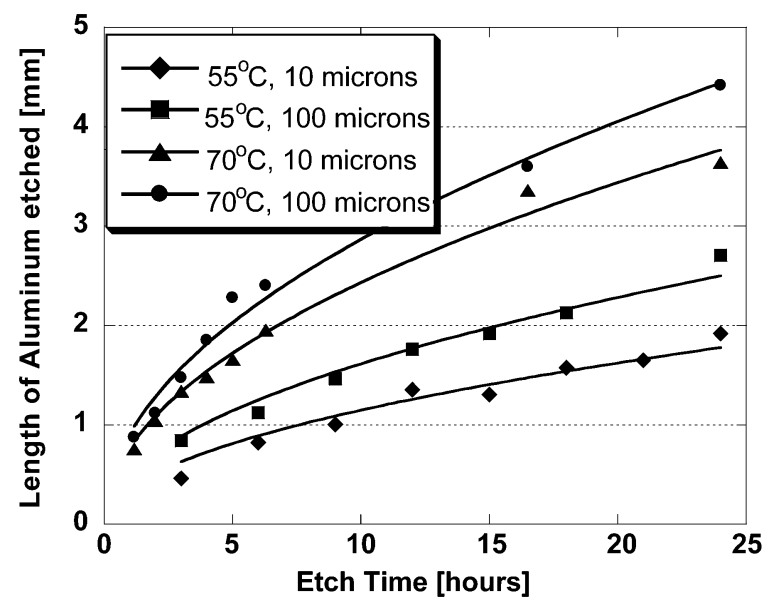

Fig. 2. Length of aluminum etched versus etch time for microchannels fabricated using aluminum as the sacrificial material. The aluminum thickness was $700 \mathrm{~nm}$ and the etch solution was (3:1) hydrochloric acid to nitric acid. The temperatures of the etchant and the width of the structure are indicated on the graph. Fits of the four data sets to (1) are shown by the solid lines on the graph.

Data are shown for structures 10 and $100 \mu \mathrm{m}$ wide and at solution temperatures of $55^{\circ} \mathrm{C}$ and $70^{\circ} \mathrm{C}$. Because the structures were cleaved twice, aluminum was removed from both sides of the structure simultaneously during etching. The numbers in the graph represent the total length of aluminum etched from both sides. The etching mechanism can be described by Deal and Grove's theory of silicon oxidation [9], and as such for sufficiently long times, the etch rate will be diffusion limited and the etch length will follow the equation:

$$
l(t) \approx \sqrt{2 k_{n} D c_{o} t}
$$

where $l(t)$ is the length of the channel etched in a given time, $k_{n}$ is a constant relating to the geometry of the channel, $D$ is the diffusion constant for etchant through the channel, and $c_{O}$ is a constant relating the concentration of the most critical component of the etch solution.

Curves with a square root dependence versus time were fit to each of the data sets in Fig. 2. The close match of the data to the curves indicates the diffusion-limited nature of the etch mechanism. The graph indicates that the speed of etching increases with temperature of the etching solution (due to an increase in the diffusion constant) and wider channels etch faster than narrow ones (due to a change in the constant $k_{n}$ related to channel geometry). Under the fastest etch conditions reported in Fig. 2, a nearly 5-mm structure can be etched in $24 \mathrm{~h}$, which is a manageable fabrication time for most devices. Total etch times can be reduced by raising the temperature of the etch solution or increasing the nitric acid concentration (increasing the constant $c_{o}$ ). Although not shown in the graph, a 2:1 hydrochloric to nitric acid mix at $85^{\circ} \mathrm{C}$ can clear a $10-\mathrm{mm}$ channel in less than $24 \mathrm{~h}$.

A mechanical finite-element analysis was done on the waveguide structure using a commercially available software package (ANSYS 6.0) to determine likely failure mechanisms and ultimate strength limits. The simulations indicated that the most likely failure mechanism was due to internal pressure caused by the release of gas during the etching mechanism. 


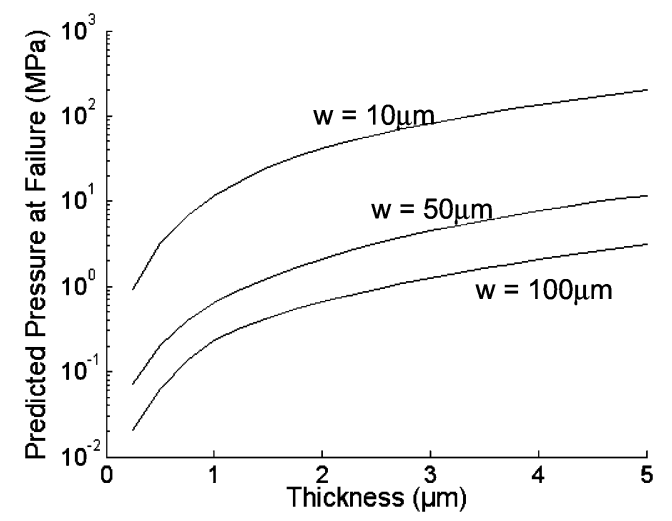

Fig. 3. Critical internal pressure for failure for microchannels predicted using finite element model. Critical pressure is plotted as a function of overcoating thickness for three different channel widths.

Using the model and the reported elastic modulus (73 $\mathrm{GPa})$ [10] and tensile strength (0.6 GPa) [11] of silicon dioxide, failure points were computed as a function of internal pressure and device geometry. In the analysis, the silicon dioxide was assumed to be homogeneous, isotropic, and linearly elastic. Predictions can be made based on the mechanical analysis for ultimate pressure the hollow waveguide structures are capable of sustaining before failure as a function of their geometry. Fig. 3 shows these predictions as a function of waveguide width and coating thickness. The graph indicates that very large pressures can be handled before failure, especially for narrow waveguides with thick coatings. The complete results of the mechanical analysis are presented elsewhere. A simple expression for this critical failure pressure $\left(P_{c}\right)$ can be derived by modeling a single beam spanning a width [12]

$$
P_{c}=2 S_{t}\left(\frac{t_{h}}{w}\right)^{2}
$$

where $S_{t}$ is the tensile strength of the overcoat material, $t_{h}$ is the thickness of the overcoat layer, and $w$ is the width of the waveguide. This simple equation reveals the functional dependence of the pressure on the width and thickness and agrees within $10 \%$ of the values calculated using the finite-element simulation when $t_{h} / w<1 / 10$.

To verify the results of the simulations and (2), structures similar to those described above were built but with a silicon dioxide top layer that varied between 250 and $2000 \mathrm{~nm}$. After etching in a $3: 1$ hydrochloric to nitric acid solution at $55^{\circ} \mathrm{C}$, the integrity of different channels was determined using an optical microscope. The experimental results are summarized in Fig. 4 for four different coating thicknesses. The graph indicates what percentage of a given microchannel was still intact after the etching process. The graph shows that for a given coating thickness, if the channel width is less than a critical width, the channel will be completely intact, but greater than this critical width, the percentage of the channel that is not collapsed falls dramatically. Also shown in the upper right-hand corner of Fig. 4 is a table showing the predicted pressure values in the structures when $50 \%$ of the channels have failed based on the $50 \%$

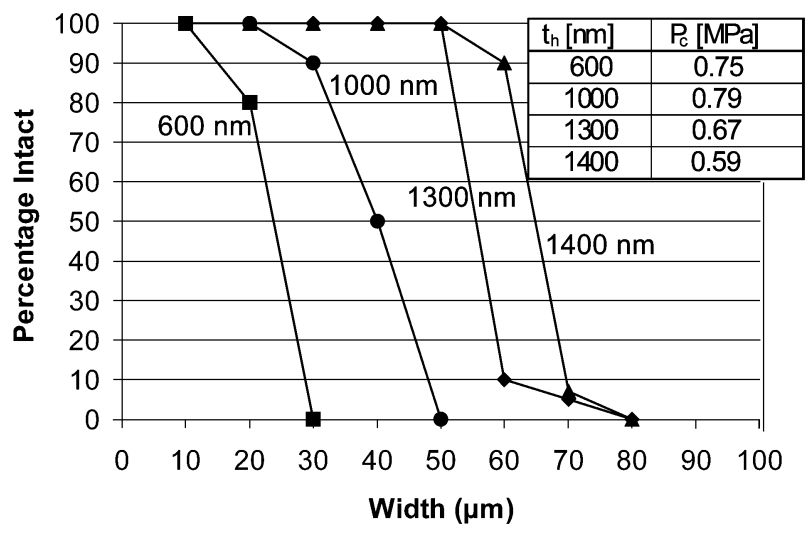

Fig. 4. Percentage of hollow microchannel intact after the etching process as a function of channel width for four different overcoating silicon dioxide thicknesses. The table in the upper right-hand corner indicates the predicted critical pressure where $50 \%$ of the channels will fail. These values were computed by using the $50 \%$ crossing points shown in the graph for the critical width and (2).

crossing points shown in Fig. 4 and (2). Assuming that the pressure created by the etching process is similar in all channels, the predicted failure pressure based on (2) should be the same for any given channel width and overcoating thickness at the critical breaking point. The values shown in the table indicate an average critical pressure of $0.70 \mathrm{MPa} \pm 16 \%$. These results are in good agreement with (2) which assumed that the pressure produced by etching is constant in tubes of different geometry, which is an approximation for the wide range of channel widths investigated here. The simulations and experiments described here indicate that our fabrication method is robust and can be applied to hollow-core waveguides filled with gases or liquids.

\section{REFERENCES}

[1] P. Yeh, Optical Waves in Layered Media. New York: Wiley, 1988.

[2] J. H. Lowry, J. S. Mendelowitz, and N. S. Subramanian, Opt. Eng., vol. 31, pp. 1982-1982, 1992.

[3] D. Yin, H. Schmidt, J. P. Barber, and A. R. Hawkins, "Integrated ARROW waveguides with hollow cores," Opt. Express, vol. 12, no. 12, pp. 2710-2715, 2004.

[4] D. Yin, J. P. Barber, A. R. Hawkins, D. W. Deamer, and H. Schmidt, "Integrated optical waveguides with liquid cores," Appl. Phys. Lett., vol. 85, pp. 3477-3479, 2004.

[5] R. Bernini, S. Campopiano, and L. Zeni, "Silicon micromachined hollow optical waveguides for sensing applications," IEEE J. Sel. Topics Quantum Electron., vol. 8, no. 1, pp. 106-110, Jan./Feb. 2002.

[6] G. R. Hadley, J. G. Fleming, and S.-Y. Lin, "Bragg fiber design for linear polarization," Opt. Lett., vol. 29, no. 8, pp. 809-811, 2004.

[7] D. Bhusari, H. A. Reed, M. Wedlake, A. M. Padovani, S. A. B. Allen, and P. A. Kohl, "Fabrication of air-channel structures for microfluidic, microelectromechanical, and microelectronic applications," J. Microelectromechanical Syst., vol. 10, pp. 400-408, 2001.

[8] M. A. Duguay, Y. Kokubun, T. Koch, and L. Pfeiffer, "Antiresonant reflecting optical waveguides in $\mathrm{SiO}_{2}-\mathrm{Si}$ multilayer structures," Appl. Phys. Lett., vol. 49, pp. 13-15, 1986.

[9] D. Westberg, O. Paul, G. I. Andersson, and H. Baltes, "Surface micromachining by sacrificial aluminum etching," J. Micromech. Microeng., vol. 6, pp. 376-384, 1996.

[10] N. Maluf, An Introduction to Microelectromechanical Systems Engineering. Norwood, MA: Artech House, 2000, pp. 17-17.

[11] T. Tsuchiya, A. Inoue, and J. Sakata, "Tensile testing of insulating thin films; humidity effect on tensile strength of $\mathrm{SiO}_{2}$ films," Sens. Actuators A, vol. 82, pp. 286-290, 2000.

[12] L. L. Howell, Compliant Mechanisms. New York: Wiley, 2001, pp. 410-410. 